\title{
Study of the possibility of increasing the probing depth by the method of reflection confocal microscopy upon immersion clearing of near-surface human skin layers
}

\author{
I.V. Meglinski, A.N. Bashkatov, E.A. Genina, D.Yu. Churmakov, V.V. Tuchin
}

\begin{abstract}
The possibility of increasing the human-skin probing depth by the method of reflection confocal microscopy (RCM) upon decreasing the amplitude of spatial fluctuations of the refractive index of the upper skin layers is considered. A change in the probing depth is estimated by analysing the spatial distribution of the probability density of the effective optical paths of detected photons calculated by the Monte Carlo method. The results of the numerical simulation are interpreted within the framework of the possible application of RCM to the study of the human skin exposed to an immersion liquid compatible to it. A diffusion of the immersion agent into the skin depth involves the equalising of the refractive indices of the structural elements of near-surface skin layers, which in turn causes a decrease in the scattering intensity and a certain increase in the transparency of the upper tissue layers. It is shown that a decrease in the light scattering in the near-surface skin layers leads to a significant increase in the probing depth obtained with the RCM technique.
\end{abstract}

Keywords: reflection confocal microscopy method, optical properties of skin, immersion-induced transparency of biological tissues, probability density of effective optical paths, localisation of optical radiation, Monte Carlo method.

\section{Introduction}

The method of reflection confocal microscopy (RCM) is widely used at present in various biomedical investigations for visualising the internal structure of biological tissues $[1,2]$. Compared to conventional microscopy methods, the main advantage of RCM is that this technique makes it possible to obtain a high-quality (with a micrometer spatial resolution) image of cellular layers. In this case, it is unnecessary to resort to a routine histological preparation

I.V.Meglinski, D.Y. Churmakov Cranfield University, School of Engineering, Cranfield, MK43 0AL, UK; e-mail:i.meglinski@cranéeld.ac.uk

A.N. Bashkatov, E.A. Genina, V.V. Tuchin N.G. Chernyshevskii Saratov State University, ul. Moskovskaya 155, 410026 Saratov, Russia; e-mail: bash@optics.sgu.ru; of the samples, which is important for in vivo studies of the cellular structure of biological objects.

A high image contrast and a high spatial resolution of RCM are achieved due to probing a small $\left(10-100 \mu^{3}\right)$ volume of the medium bounded by the size of the central focal spot, which is formed by a focusing optical system. The localisation of a desired signal within the measured volume of such small dimensions becomes possible as a result of mutual optical matching between the laser radiation source, the measured volume, and the photodetector. The fields of vision of the laser source and photodetector are limited by diaphragms of submicrometer dimensions, which are mounted in the planes of objects and images (Fig. 1). If the penetration depth $z_{\mathrm{f}}$ of the lens focus into the medium does not exceed three - four lengths of the mean photon free path $l_{\text {ph }}$ [3], then such diaphragming (see Fig. 1) ensures that the photons reflected strictly back by the structural elements of the probed microscopic volume (so-called ballistic photons) dominate in the detected signal. As is known, it is these pho-tons that carry valid information that allows one to reconstruct the internal structure of the medium under study [4].

As $z_{\mathrm{f}}$ increases, i.e., when the focus penetrates deeper into the scattering medium, the fraction of ballistic photons in the detected signal decreases, while the fraction of photons scattered by the medium increases. Under typical conditions of experiments with biological tissues, the RCM technique allows one to distinguish ballistic photons against a background of the totality of the medium-scattered photons, until $z_{\mathrm{f}}$ becomes higher than $l_{\mathrm{ph}}$ by a factor of $5-8$ [5]. In other words, because of an intense multiple light scattering characteristic of most of biological tissues [6], the RCM method makes it possible to obtain an image of the cellular structure of skin tissues located at a depth of at most $300-400 \mu \mathrm{m}[2,7]$.

It is obvious that, using the RCM method, the maximum scanning depth can be achieved by increasing $l_{\mathrm{ph}}$ or (which is the same) reducing the multiplicity of scattering in the upper layers of the probed medium (human skin in our case). Scattering in skin tissues can be suppressed for a short time by applying solutions of glucose, glycerine, and some other biologically compatible immersion liquids to the skin surface or injecting them into the skin derma [8-12]. It should be emphasised that the use of glycerine and propylene glycol as immersion liquids allows one to obtain a much higher image contrast and a larger skin probing depth by the method of low-coherence optical tomography [13]. Similar results were obtained for whole blood [14, 15]. However, despite numerous experimental data [10-15] objectively 


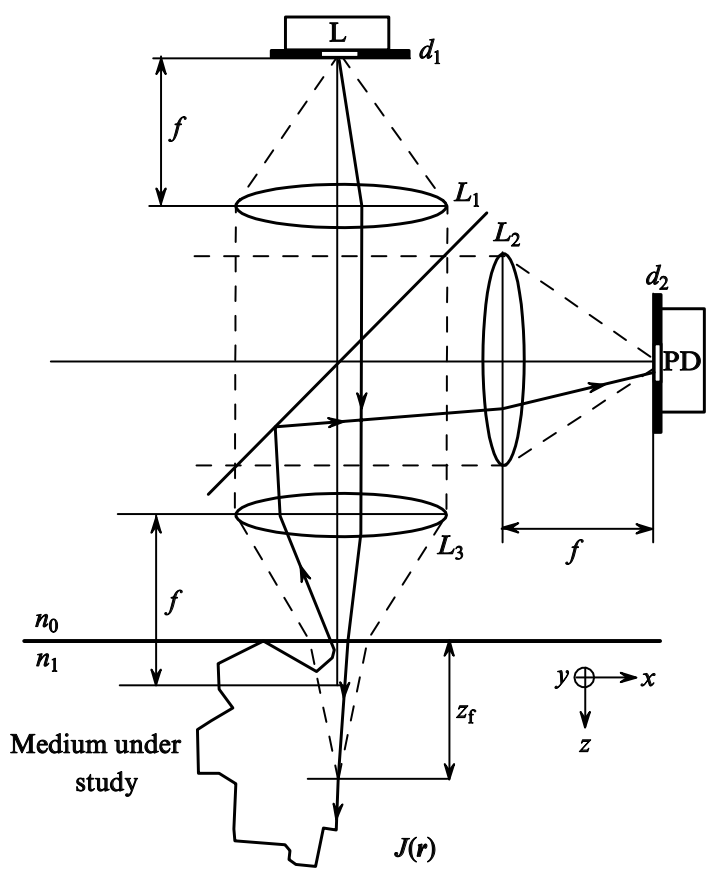

Figure 1. Schematic of the confocal probing method: $\left(L_{1}, L_{2}, L_{3}\right)$ shortfocus lenses $(f=8 \mathrm{~mm})$ forming in pairs a small-aperture collimator; $\left(d_{1}, d_{2}\right)$ limiting diaphragms with diameters of $10 \mu \mathrm{m} ;\left(z_{\mathrm{f}}\right)$ depth of focal point immersion into the medium; (L) laser source; (PD) detector of optical radiation; and $\left(n_{0}, n_{1}\right)$ refractive indices of the external and studied media.

testifying that the optical immersion method is promising as applied to biological tissues, reliable quantitative data on the skin probing depth under the conditions of immersioninduced transparency are currently required.

This study is aimed at the analysis of the possibility of increasing the effective depth of probing biological tissues by the RCM method by decreasing the scattering coefficient in them upon their optical immersion.

\section{Probability density function of the effective optical paths}

Due to multiple scattering, the radiation inside a biological tissue becomes distributed within a certain region $G[6,8]$. The features of the spatial distribution of radiation in this region are determined by the geometrical and optical properties of the medium under study and the conditions of its illumination (i.e., by the profile of the incident beam, the angle of its incidence on the medium surface, etc.) $[16,17]$. The optical parameters of the medium used in our analysis are as follows: $\mu_{\mathrm{s}}$ is the scattering coefficient, $\mu_{\mathrm{a}}$ is the absorption coefficient, $g$ is the anisotropy factor, and $n$ is the refractive index. The geometrical properties are determined by the internal structural features of the medium: the interfaces of layers, their shapes and depths, and the degree of the surface roughness.

From the practical viewpoint, the region $G$ and the features of the radiation distribution in it are of interest for optical dosimetry problems [6]. In optical diagnostics and tomography problems, the effective measurement region $J$, or the so-called measured volume (Fig. 2), is of greatest interest. This is explained by the fact that the analysis of the

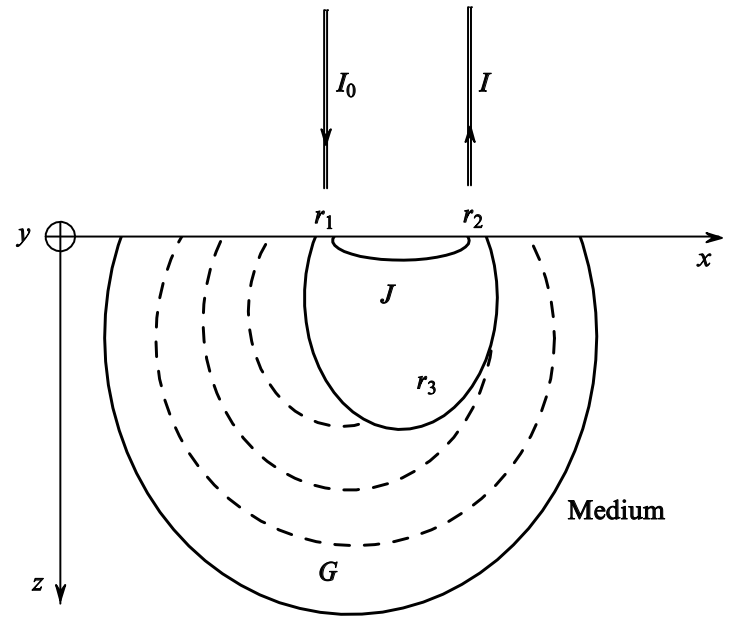

Figure 2. Schematic of the region $G$ of spatial distribution of the incident radiation in a randomly inhomogeneous multilayer medium with intense light scattering exposed to fiber-optical probing: $(J,(J \subset G)$ region of spatial distribution of the effective photon optical paths, which characterises the detected-signal localisation region; $\left(r_{1}\right)$ coordinate of the photon packet entrance to the medium; $\left(r_{2}\right)$ coordinate of the photon packet exit from the medium; and $\left(r_{3}\right)$ coordinate of the medium under study.

effective measurement region $J$ makes it possible to reliably determine the optimal configuration of an optical system intended for probing the reference volume located at a specified depth in the medium under study.

At present, direct measurements of the region $J$ in biological tissues can evidently be performed only in vitro [17] and only if $J$ coincides with the region $G$ of distribution of the incident radiation $G(J \equiv G)$ or, in other words, only when the entire surface of the medium under study serves as a photodetector. This can be implemented in practice, for example, when detecting the radiation diffusely back reflected by an integrating sphere, whose working window has a diameter much larger than the size of the radiation source (a collimated laser beam). Any diaphragming of the detector field of vision (e.g., by an optical fibre) significantly restricts the measured volume $J$ (Fig. 2): $J<G, J \subset G$. In this case, the $J$ profile can be determined only by numerical methods, for example, by analysing the temporal pointspread function, which characterises the mean time of localisation of the photons detected by the photodetector within a certain elementary volume of the probed medium.

Taking into account the fact that the RCM method uses continuous laser radiation, according to [18], we change from the analysis of a temporal point-spread function to considering the effective optical paths $L$ of the detected photons and to the analysis of the probability density function of the effective optical paths $J(\boldsymbol{r})$. Hereafter, the path that a photon has travelled directly in the medium between the source and detector is assumed to be the effective optical path. This allows us to determine the photon path length and/or the time of photon presence in each elementary volume of the medium, which in turn determines the possibility of considering $J(\boldsymbol{r})$ instead of the temporal point-spread function [18]. For the methods based on measurements of the backscattered radiation intensity, we specify, according to [18-20], $J(\boldsymbol{r})$ for a homogeneous intensely scattering medium in the form 


$$
J\left(r_{1}, r_{2}, r_{3}\right)=\frac{\partial F\left(r_{1}, r_{2}\right)}{\partial \mu_{\mathrm{a}}\left(r_{3}\right)},
$$

where $\partial \mu_{\mathrm{a}}\left(r_{3}\right)$ is the change in the medium absorption coefficient at the point $r_{3} ; r_{1}$ is the point determining the position of the radiation source on the medium surface; and $\partial F\left(r_{1}, r_{2}\right)$ is the change in the radiation flux, which is detected at the point $r_{2}$ at the medium surface and is defined as

$$
F\left(r_{1}, r_{2}\right)=-\ln \left[\frac{I\left(r_{2}\right)}{I_{0}\left(r_{1}\right)}\right] .
$$

Here, $I_{0}\left(r_{1}\right)$ is the incident radiation intensity at the point $r_{1}$ and $I\left(r_{2}\right)$ is the radiation intensity at the point $r_{2}$ in the detector field of vision. We assume that, for nonmodulated continuous incident radiation, the quantities $I\left(r_{2}\right)$ and $I_{0}\left(r_{1}\right)$ are related by the Bouguer-Lambert-Beer law: $I\left(r_{2}\right)=$ $I_{0}\left(r_{1}\right) \exp \left(-\mu_{\mathrm{a}} L\right)$. By substituting (2) into (1), we obtain

$$
J\left(r_{1}, r_{2}, r_{3}\right)=-\frac{1}{I\left(r_{2}\right)} \frac{\partial I\left(r_{2}\right)}{\partial \mu_{\mathrm{a}}\left(r_{3}\right)} .
$$

Thus, $J\left(r_{1}, r_{2}, r_{3}\right)$ determines the specific radiation flux which is registered by the detector within the bounds of the elementary volume $(\Delta x \times \Delta y \times \Delta z)$ of the medium located at the point $\left(\forall r_{3} \in G\right)$. The function $J(r)$ characterises the complete spatial distribution of the specific radiation flux detected in the medium and is determined by the distribution of the effective optical paths (see Fig. 2). In the case of a complex inhomogeneous multiplayer medium, whose optical properties change from layer to layer, let us consider partial components of the effective optical paths separately for each $i$ th layer:

$$
J(\boldsymbol{r})=-\frac{1}{I} \frac{\partial I}{\partial \mu_{\mathrm{a} i}} .
$$

Note that a similar problem in one or another form was solved earlier in the application to near IR (optical) tomography of biological objects [18-23]. This accounts for a variety of terms used to define $J(\boldsymbol{r})$ : photon measurement density function $[19,20]$, sensitivity region [21], photon sampling volume [22], photon hitting density [23], spatial sensitivity profile [24], etc. In our opinion, the term 'the function of probability density of the effective optical paths' is the most suitable definition for $J(\boldsymbol{r})$.

\section{The use of the Monte Carlo method for calculating the probability density of the effective optical paths during confocal probing of biological skin tissues}

The complexity of the internal structure of human skin, which represents a random multilayer medium strongly scattering and absorbing optical radiation, does not allow us to calculate $J(\boldsymbol{r})$ by the methods based on the diffusion approximation [22-25]. For the same reason, the methods of simple and double local estimates turn out to be inadequate for solving this problem $[26,27]$. The geometry of the confocal probing system (see Fig. 1) combined with complex configurations of the interfaces between the medium layers substantially narrows the applicability of the finite element method $[19,20]$ and the photon random walk method [28], because these methods cannot adequately take into account the degree of probe radiation focusing and the total internal reflection at the layer interfaces in the simulation of the propagation of the focused radiation in the multilayer medium. In this case, the Monte Carlo (MC) numerical simulations $[26,29,30]$ is, in our opinion, the most optimal technique for determining the probability density of the effective optical paths.

In this study, we use a step-by-step simulation algorithm $[29,30]$ modified for the confocal probing system (Fig. 1). Within the framework of this algorithm, the surfaces of the layer interfaces are represented in the form of randomly periodic wavy surfaces. The heights of peaks for each surface are selected so as to ensure their best correspondence to the shapes of the layer interfaces, which are inherent in structural geometrical features of human skin [2, 31, 32]. The degree of surface roughness is chosen in accordance with typical statistically average sizes of cells and (or) collagen fibres characteristic of different layers of human skin $[2,31,32]$. It has been shown that the consideration of the roughness of the layer interfaces is important in the simulation of laser probing of a multilayer medium by a focused narrow beam [33]. It has also been shown that, despite an intense multiple light scattering by layers of the medium under study, if the distance between the source and detector is obviously small, the formation of the spatial distribution region $J(\boldsymbol{r})$ depends on the geometrical features of the medium [17].

In addition to an improved geometrical model of human skin, another essentially important distinguishing feature of the method $[29,30]$ is the rejection of the 'roulette' procedure, which is applied in the currently popular version of the MC method [16, 34-36]. This procedure is intended for equalising the energy balance between the incident radiation and the radiation that was absorbed by the medium and escaped from the analysed region due to scattering. The implementation of the roulette procedure consists in a periodic increase in the statistical weight of separate randomly selected photon packets instead of the total statistical weight of the group of photon packets 'lost' in the medium (i.e., the photons that crossed the boundaries of the region of interest and emerged from it or those excluded from the further consideration as a consequence of their small statistical weight). Thus, an increase in the statistical weight of an individual photon raises its chances for 'survival' (i.e., a successful emergence from the medium at a given point). However, in this case, an unjustified increase in the photon optical path length takes place, which obviously leads to erroneous results in calculations of the distribution of the effective photon optical paths using the roulette procedure [16, 34-36].

In the context of the algorithm of the MC method $[29,30]$ used in this work, the effective photon optical paths were calculated using the method of calculation of true trajectories [37]. In this method, the simulation of the trajectory of each individual photon is described in terms of direction cosines. The initial conditions for the injection of photon packets into the medium and their detection conditions for a specified confocal probing system are based on the ray optics approach $[3,38]$. The trajectory of a photon packet is tracked, until the statistical weight of the packet, which decreases proportionally to the Fresnel reflection coefficients (refractive index) as a result of reflection and refraction processes occurring at the medium 
surface, becomes below 0.001 . This allows us to take into account the total internal reflection at the medium surface. The trajectories of all photon packets that reached the detection region are registered. In this work, we restrict ourselves to the detection of $5 \times 10^{6}$ photon packets.

Then, following the trajectory of each individual packet, we take into account the absorption of optical radiation in the medium, as was done in $[29,30]$; i.e., we reduce the current value of the packet weight proportionally to $\exp \left(-\mu_{\mathrm{a}} l_{\mathrm{ph}}\right)$ at each step of its path. Such a combination of the true trajectory calculation method with the use of statistical weights makes it possible to comparatively easily calculate the probability density of the effective optical paths (4) for an arbitrary medium probing geometry and an arbitrary set of optical parameters of the medium layers.

\section{Results and discussion}

Fig. 3 shows the results of numerical simulation of the function $J(\boldsymbol{r})$ performed by the method described above for a confocal probing scheme with a focus of the objective lens immersed into a homogeneous scattering medium to a depth of $300 \mu \mathrm{m}$. The parameters of the confocal probing scheme (Fig. 1) used in calculations were selected as one of possible versions corresponding to the objective lenses used in RCM [1,2]. The choice of medium optical characteristics for the cases presented in Fig. 3 is based on the experimental data [3] that determine the limiting scanning depth for RCM. As was expected, when the immersion depth $z_{\mathrm{f}}$ of the lens focus does not exceed 3-4 mean photon free path lengths $\left(\mu_{\mathrm{s}} \leqslant 10 \mathrm{~mm}^{-1}\right.$ or, equivalently, $\left.l_{\mathrm{ph}} \geqslant 100 \mu \mathrm{m}\right)$, clearly pronounced photon focusing at a depth of $300 \mu \mathrm{m}$ is observed in the spatial distribution of the density of effective optical photon paths (Fig. 3a).

If $z_{\mathrm{f}}$ amounts to $8-20$ mean photon free path lengths in the medium ( $\mu_{\mathrm{s}}=26.6-40 \mathrm{~mm}^{-1}$ and, correspondingly, $\left.l_{\mathrm{ph}}=25-37.5 \mu \mathrm{m}\right)$, then the tendency of probing-radiation focusing in the medium is held (Fig. 3b). However, the central focal spot region is much larger than in the case illustrated by Fig. 3a, which affects the quality of the image obtained. With a further increase in the medium scattering coefficient $\left(\mu_{\mathrm{s}} \geqslant 100 \mathrm{~mm}^{-1}\right)$ and the corresponding shortening of the mean photon free path $\left(l_{\mathrm{ph}} \leqslant 1 \mu \mathrm{m}\right)$, the incident radiation turns out to be defocused, although its narrow directivity remains unaltered (Fig. 3c).

These data (Fig. 3) clearly illustrate the possibility of localising the focused probing laser radiation inside a homogeneous, multiply scattering, anisotropic $(g=0.9)$ medium, which is probed by the RCM technique and weakly absorbs optical radiation $\left(\mu_{\mathrm{a}}=0.01 \mathrm{~mm}^{-1}\right)$, and are in good agreement with the experimental data and the results obtained by independent simulation methods $[27,38$, 39].

An inhomogeneous distribution of the medium optical characteristics in depth significantly affects the distribution of the effective photon optical paths in a multilayer medium, such as, for example, human skin. The results of $J(\boldsymbol{r})$ calculations shown in Fig. 4 clearly demonstrate a collapse of the narrow focused $\left(z_{\mathrm{f}}=300 \mu \mathrm{m}\right)$ laser beam due to the effects of intense light scattering in the medium layers and the roughness of their interfaces and the rate of this process. The optical properties of the medium layers (see Table 1) used in our simulation correspond to the tissues of the normal Caucasian-type human skin in the near IR region
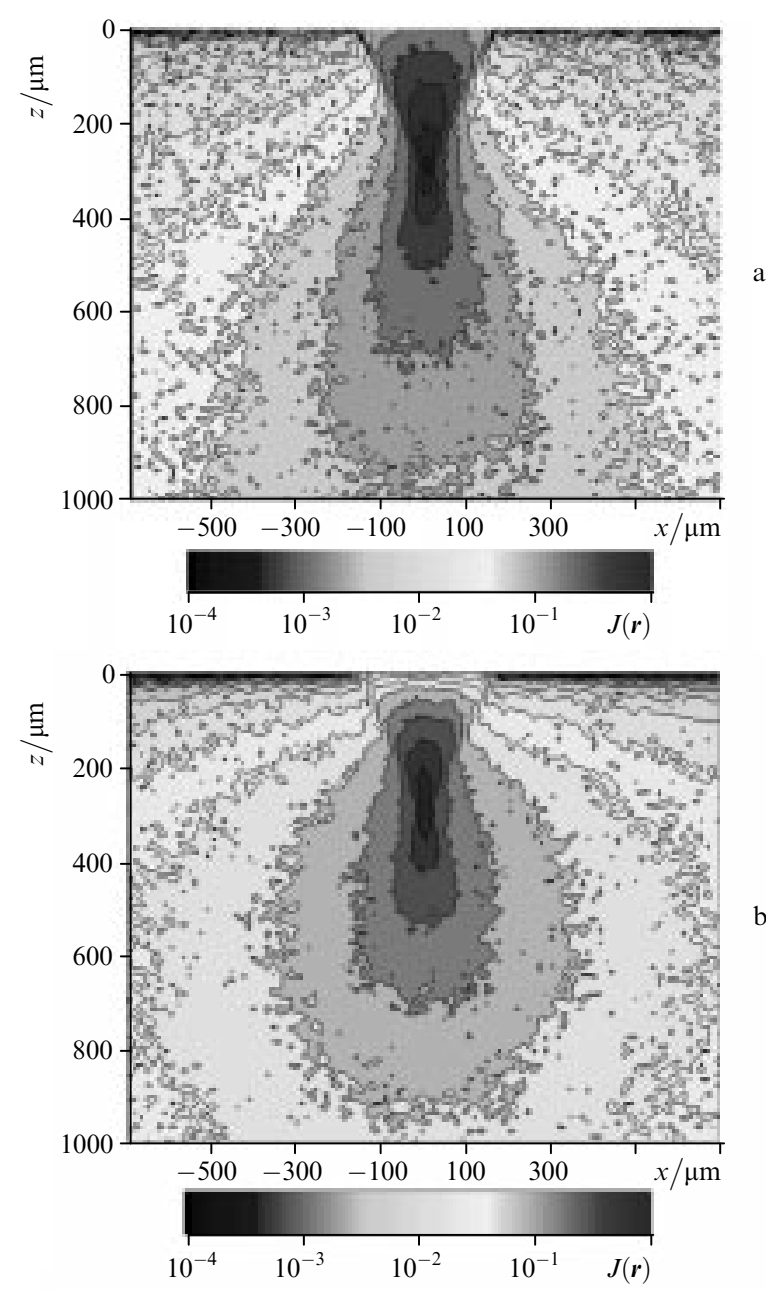

$\mathrm{b}$

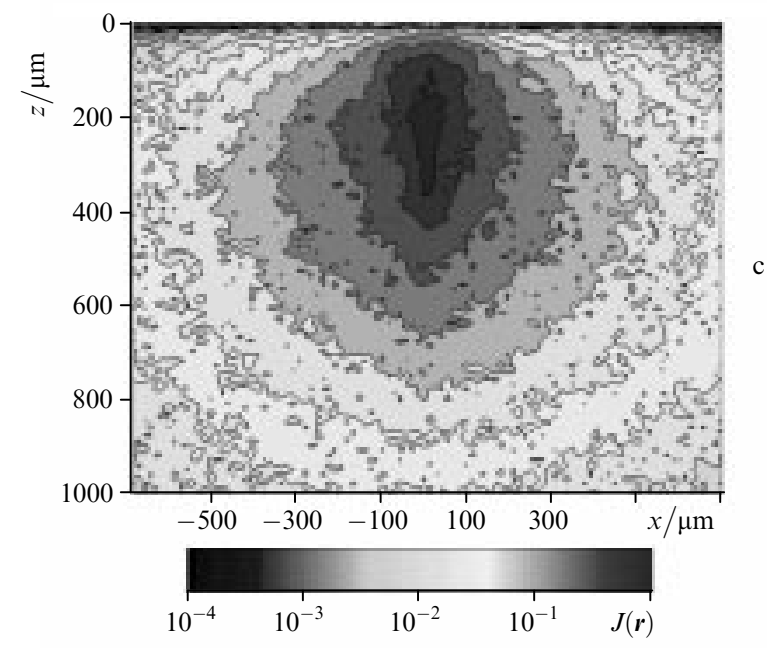

Figure 3. Spatial distribution $J(\boldsymbol{r})$ of the probability density of the effective photon optical paths calculated for an intensely scattering $\left(\mu_{\mathrm{s}}=10\right.$ (a), 26.6 (b), and $100 \mu \mathrm{m}^{-1}$ (c)), homogeneous $(n=1.4)$, anisotropic $(g=0.9)$, and absorbing $\left(\mu_{\mathrm{a}}=0.01 \mathrm{~mm}^{-1}\right)$ medium. The focal point immersion depth is $z_{\mathrm{f}}$.

$(\lambda=600-700 \mathrm{~nm})[6]$. The filling of tissues with blood, the degree of blood flow oxygenation, the hematocrite level, and the contents of water and melanin were taken into account similarly to the approach of [40], which allows for a sufficiently accurate simulation of fragments of the reflection spectra. 


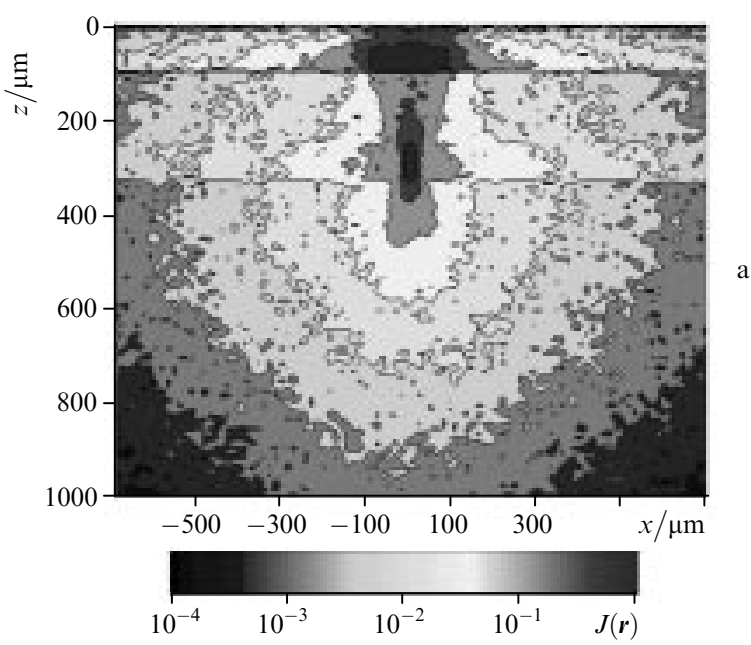

$(2.56 \pm 0.13) \times 10^{-6} \mathrm{~cm}^{2} \mathrm{~s}^{-1}$ [11]. The change in the refractive index of the intratissue liquid in skin layers was calculated according to the Gladstone-Dahl law [44]. The scattering coefficients of separate skin layers were calculated using the Mie theory [45]. The values of the optical parameters listed in Table 2 and utilised in this simulation represent the state of skin tissues in 20 and $40 \mathrm{~min}$ after the deposition of the glucose solution onto the skin surface. They agree well with the experimental data $[10-13,46]$. Note that, since the diffusion coefficients for low-molecular compounds are rather close to each other, the data in Table 2 reflect changes in the scattering characteristics of various skin tissue layers when using not only a glucose aqueous solution as an immersion agent but also other immersion liquids with refractive indices of approximately 1.39. Aqueous solutions of glycerine, propylene glycol, etc. can be used as immersion agents.

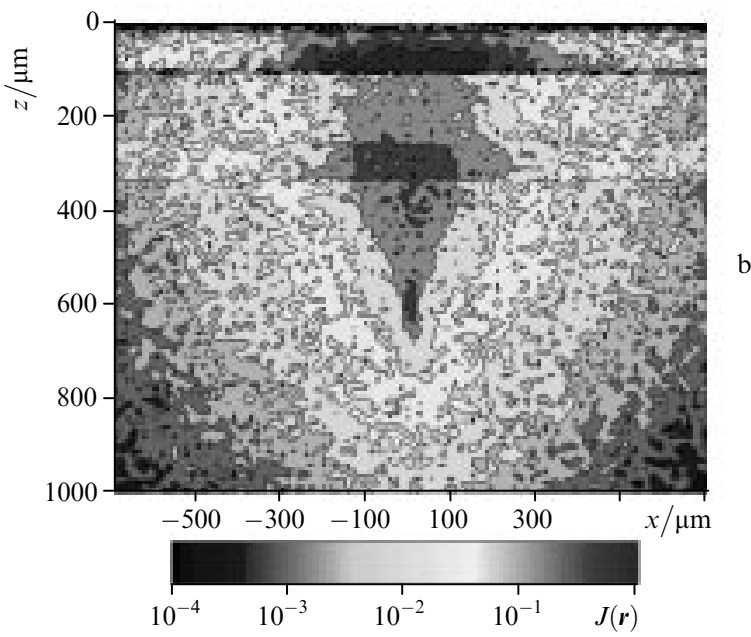

Figure 4. Spatial distribution $J(\boldsymbol{r})$ of the probability density of the effective photon optical paths in a multilayer medium calculated for $z_{\mathrm{f}}=300$ (a) and $600 \mu \mathrm{m}$ (b). The optical properties of medium layers are listed in Table 1.

Since the cellular structure of the epidermis is dense [41], the substance diffusion rate through it is two-three orders of magnitude lower than through the derma with a fibrosis structure [31,32]. In this study, changes in the scattering coefficient (in the concentration of the immersion agent in skin layers) are quantitatively described using a model which assumes a constancy of the diffusion coefficient in a given skin layer [42, 43]. For example, the diffusion coefficient for a $40 \%$ aqueous solution of glucose in derma is

Table 1. Table 1. Optical properties of skin layers used in the simulation.

\begin{tabular}{|c|c|c|c|c|c|}
\hline Layer & $\mu_{\mathrm{s}} / \mathrm{mm}^{-1}$ & $\mu_{\mathrm{a}} / \mathrm{mm}^{-1}$ & $g$ & $n$ & Thickness/mm \\
\hline Corneous layer & 100 & 0.1 & 0.8 & 1.5 & 20 \\
\hline Epidermis & 45 & 0.15 & 0.8 & 1.34 & 80 \\
\hline $\begin{array}{l}\text { Derma with } \\
\text { capillary loops }\end{array}$ & 30 & 0.068 & 0.9 & 1.39 & 150 \\
\hline $\begin{array}{l}\text { Derma with surface } \\
\text { vessel plexus }\end{array}$ & 25 & 0.095 & 0.95 & 1.4 & 80 \\
\hline Derma & 20 & 0.073 & 0.76 & 1.39 & 1500 \\
\hline $\begin{array}{l}\text { Derma with deep } \\
\text { vessel plexus }\end{array}$ & 30 & 0.118 & 0.95 & 1.4 & 100 \\
\hline Hypodermic fat & 15 & 0.068 & 0.8 & 1.44 & 5000 \\
\hline
\end{tabular}

Table 2. Optical properties of human skin layers used in the numerical simulation of an immersion-induced transparency of the skin tissue.

\begin{tabular}{|c|c|c|c|c|c|c|}
\hline \multirow{2}{*}{ Layer } & \multicolumn{2}{|c|}{$\mu_{\mathrm{s}} / \mathrm{mm}^{-1}$} & \multirow{2}{*}{$\mu_{\mathrm{a} /} \mathrm{mm}^{-1}$} & \multirow{2}{*}{$g$} & \multicolumn{2}{|c|}{$n$} \\
\hline & $20 \mathrm{~min}$ & $40 \mathrm{~min}$ & & & $20 \mathrm{~min}$ & $40 \mathrm{~min}$ \\
\hline Corneous layer & 70 & 50 & 0.10 & 0.8 & 1.47 & 1.45 \\
\hline Epidermis & 15 & 5 & 0.15 & 0.8 & 1.36 & 1.36 \\
\hline $\begin{array}{l}\text { Derma with } \\
\text { capillary loops }\end{array}$ & 20 & 15 & 0.068 & 0.9 & 1.4 & 1.4 \\
\hline $\begin{array}{l}\text { Derma with } \\
\text { surface vessel } \\
\text { plexus }\end{array}$ & 20 & 15 & 0.095 & 0.05 & 1.4 & 1.4 \\
\hline Derma & 20 & 20 & 0.073 & 0.76 & 1.39 & 1.39 \\
\hline $\begin{array}{l}\text { Derma with } \\
\text { deep vessel ple } \\
\text { xus }\end{array}$ & & 30 & 0.118 & 0.95 & 1.4 & 1.4 \\
\hline
\end{tabular}

An interesting fact is that the upper medium layers, which are characterised by the most intense light scattering and absorption (see Table 1), exert a certain screening effect on the focusing region (see Fig. 4a). Although, in this case, the probability density of the effective optical paths in the second layer (epidermis) has a smaller absolute value, it is still comparable with $J$ in the region of the central focal spot (Fig. 4a). When the depth of the focal point immersion into the medium is doubled $\left(z_{\mathrm{f}}=600 \mu \mathrm{m}\right)$, the $J$ value in the region of the central focal spot considerably decreases and becomes lower than in the screening region (Fig. 4b). In this case, the fraction of the signal from the fourth medium layer, which, within the framework of our model, represents a region of derma with a surface vessel plexus and is characterised by higher scattering and absorption coefficients than in adjacent layers (see Table 1).

As the scattering coefficients $\mu_{\mathrm{s}}$ of the two upper medium layers decrease, the depth of penetration of a narrow focused optical beam into the medium significantly increases (Fig. 5). If the radiation is focused at a depth of $300 \mu \mathrm{m}$, the screening effect of the upper layers disappears (see Fig. 5a). When the radiation is focused at a depth of $600 \mu \mathrm{m}$ (see Fig. 5b), the maximum $J(r)$ values in the region of the derma layer with a surface vessel plexus and at the location of the central focal spot are comparable, as is in the case shown in Fig. $4 \mathrm{~b}$.

A further decrease in the scattering coefficients of the upper derma layers from 20 to $15 \mathrm{~mm}^{-1}$ (see Table 2) 

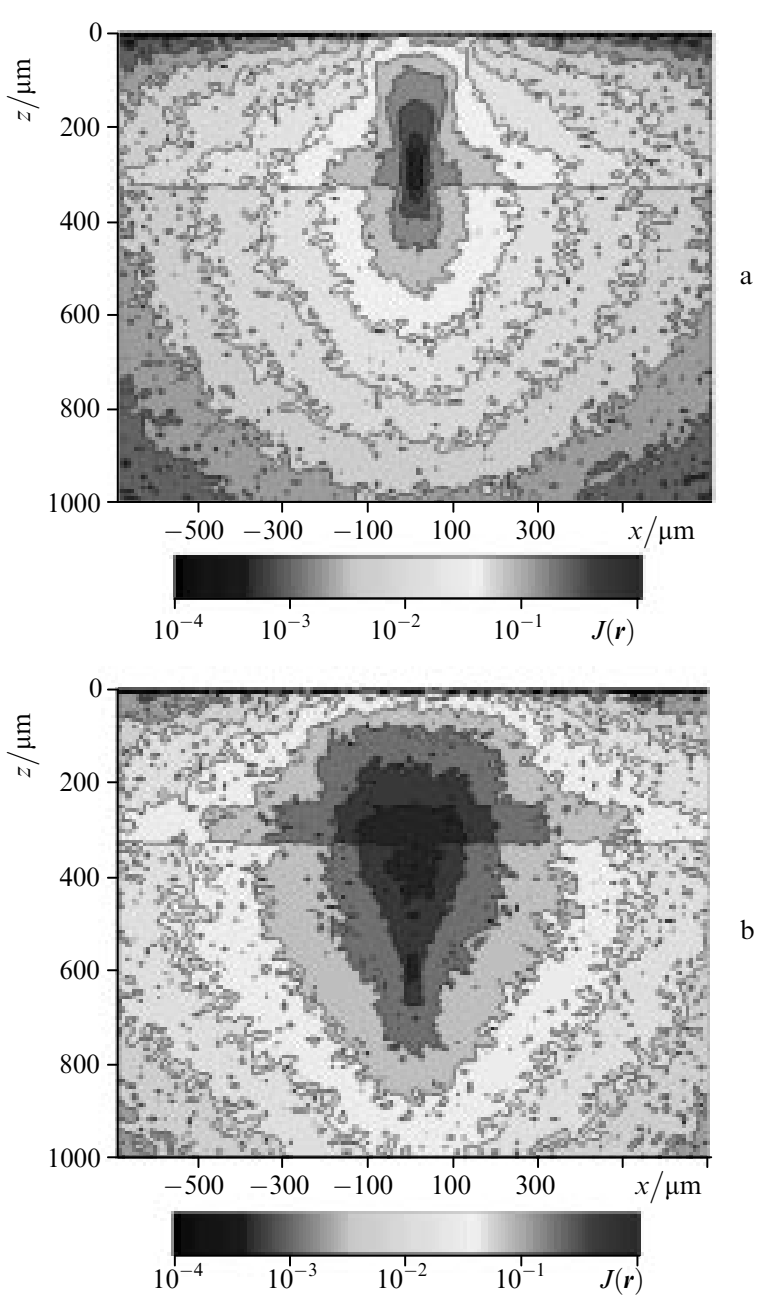

Figure 5. Spatial distribution $J(\boldsymbol{r})$ of the probability density of the effective photon optical paths in the case of 20-min immersion-induced transparency of skin calculated for $z_{\mathrm{f}}=300$ (a) and $600 \mu \mathrm{m}$ (b). The optical properties of the medium layers are listed in Table 2.

appreciably improves the spatial resolution in the region of the central focal spot at $z_{\mathrm{f}}=300$ and $600 \mu \mathrm{m}$ (Fig. 6). Moreover, if the radiation is focused to a depth of even $900-1000 \mu \mathrm{m}$, a clearly pronounced localisation of the effective photon optical paths is observed in the region of the central focal spot (Fig. 7). An improvement of the spatial resolution of the RCM method, in particular, the removal of the screening effect of the upper skin layers upon an immersion-induced increase in their transparency, makes it possible to significantly increase the possibilities of optical diagnostics of malignant tumours in skin tissues.

Note that, when a significant decrease in the skin scattering coefficient is necessary, an intradermal injection of an immersion agent is required. Fig. 8 shows a typical time dependence of the reflectance for human skin at a wavelength of $700 \mathrm{~nm}$ under the action of an injection of a $40 \%$ glucose solution. A substantial decrease in the reflectance with time is determined by the diffusion of glucose into the biological tissue and a decrease in the scattering coefficient accompanying this process. It should be noted that such a significant, approximately threefold decrease in the skin reflectance is associated with the injection of an immersion agent directly into the skin derma, because derma has the maximum thickness among other
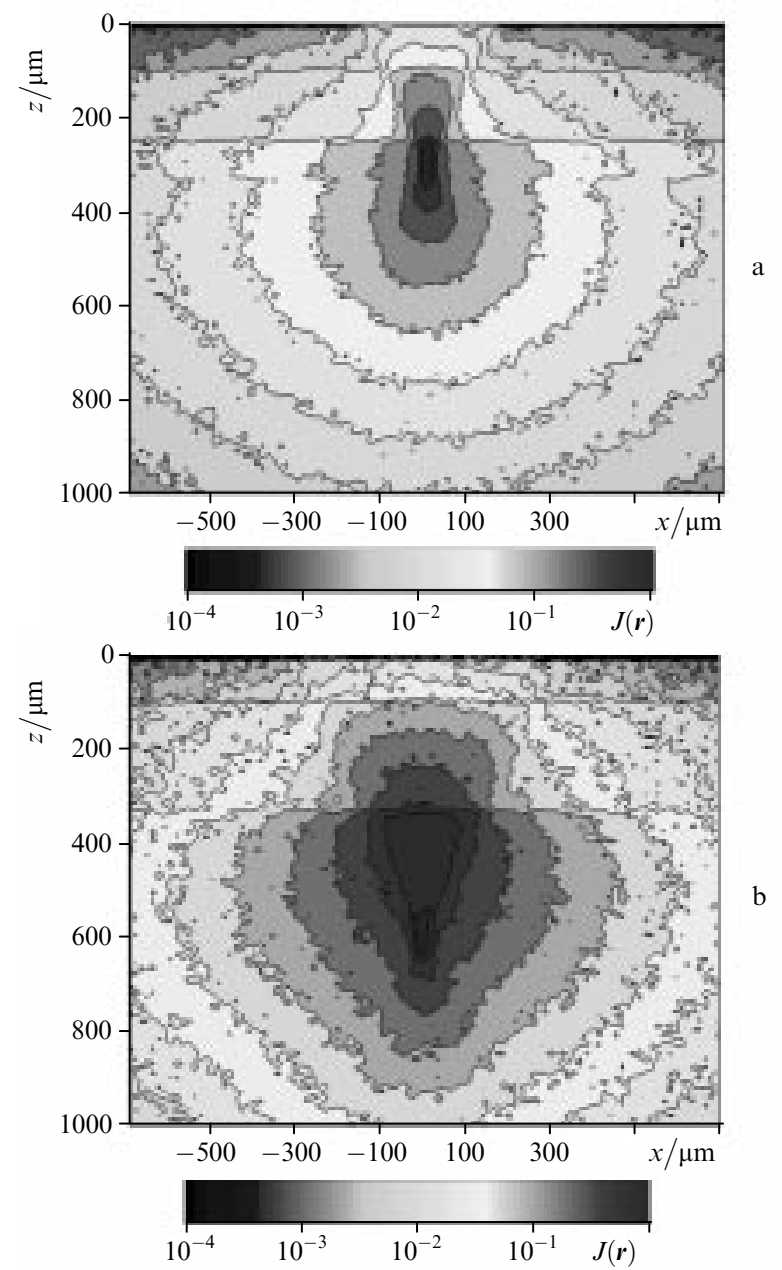

Figure 6. Spatial distribution $J(\boldsymbol{r})$ of the probability density of the effective photon optical paths in a multilayer medium calculated for the case of 40-min immersion-induced transparency of skin for $z_{\mathrm{f}}=300$ (a) and $600 \mu \mathrm{m}(\mathrm{b})$. The optical properties of the medium layers are listed in Table 2.

skin tissue layers and the skin reflection spectrum forms mainly due to the derma scattering characteristics. Calcu-

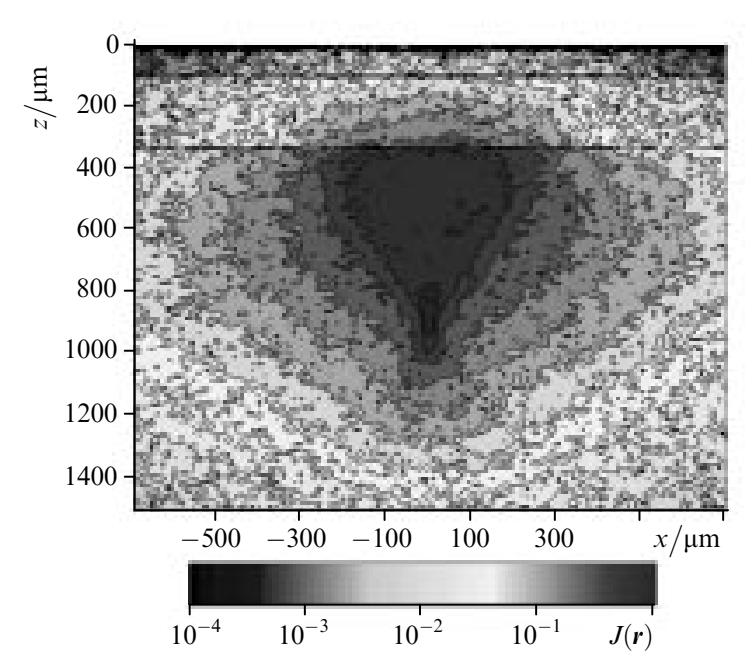

Figure 7. Spatial distribution $J(\boldsymbol{r})$ of the probability density of the effective photon optical paths in a multilayer medium calculated for $z_{\mathrm{f}}=900 \mu \mathrm{m}$. 


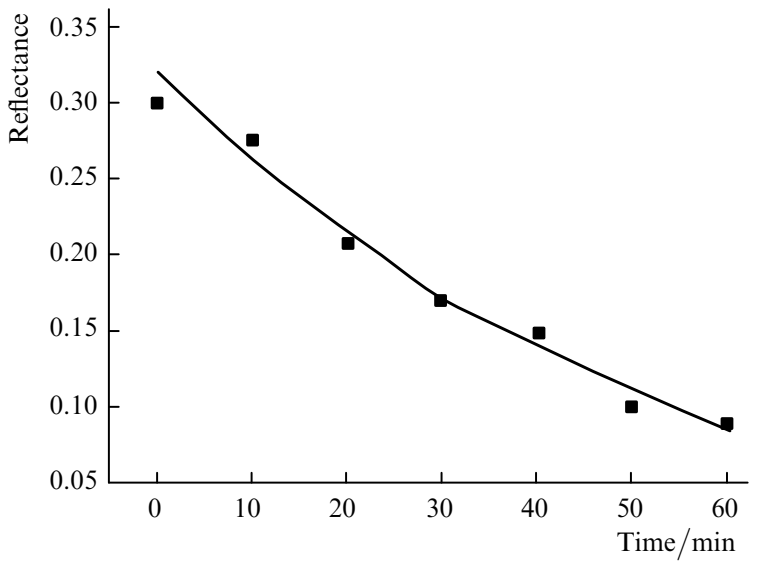

Figure 8. Time dynamics of the in vivo human-skin reflection coefficient at $\lambda=700 \mathrm{~nm}$ under intradermal injection of a $40 \%$ glucose solution.

lations based on the Mie theory show that the complete replacement of the skin intratissue liquid by an immersion liquid with a refractive index of 1.39 leads to an approximately twofold reduction of the skin scattering coefficient. Hence, despite the fact that, compared to the intradermal injection, the deposition of immersion agents onto skin is a simpler operation preferable from the medical viewpoint, the injection of an immersion agent into skin ensures a significantly higher optical immersion efficiency (the image depth and quality), which is an essential factor in the early diagnostics of skin cancer.

\section{Conclusions}

We have performed a numerical simulation of the probability density of the effective optical paths in homogeneous and multilayer media, which are characterised by strong light scattering and absorption, upon their confocal probing. The numerical simulation was performed by the MC method [16, 17, 40] modified for confocal probing. The optical properties of the model-medium layers are associated with the optical properties inherent in normal human skin tissues, including the case of their immersion-induced transparency. The results obtained clearly demonstrate that, as the scattering intensity in the upper layers of the medium decreases, the RCM probing depth increases by a factor of $3-4$. These results are in good agreement with the experimental data [10-13,41,42], allowing one to interpret them within the framework of the application of RCM to human skin studies, when the skin surface is exposed to solutions of osmotically active immersion substances.

Acknowledgements. This work was supported by the Russian Foundation for Basic Research ('Leading Scientific Schools', Grant No. 00-15-96667), U.S. Civilian Research \& Development Foundation for the Independent States of the Former Soviet Union No. REC-006, and supported in part by the Russian Foundation for Basic Research (Grant No. 89433).

\section{References}

1. Wilson T. Confocal Microscopy (San Diego: Academic Press, 1990) p. 426
2. Pawley J.B. Handbook of Biological Confocal Microscopy (New York: Plenum Press, 1995) p. 632.

3. Schmitt J.M., Knuettel A., Yadlowsky M. J. Opt. Soc. Am. A, 11, 2226 (1994).

4. Lyubimov V.V. Opt. Spektrosk., 76, 814 (1994).

5. Izatt J.A., Hee M.R., Owen G.M., Swanson E.A., Fujimoto J.G. Opt. Lett., 19, 590 (1994).

6. Tuchin V.V. Lazery $i$ volokonnaya optika v biomeditsinskikh issledovaniyakh (Lasers and Fiber Optics in Biomedical Investigations) (Saratov: Izd. Saratov Univer., 1998).

7. Rajadhyaksha M., Zavislan J.M. Clinical \& Experimental Techniques, 14, 26 (1998).

8. Tuchin V.V. Usp. Fiz. Nauk, 167, 517 (1997).

9. Hatcher M. Opto \& Laser Europe, 10, 37 (2001).

10. Vargas G., Chan E.K., Barton J.K., Rylander III H.G., Welch A.J. Laser in Surgery and Medicine, 24, 133 (1999).

11. Tuchin V.V., Bashkatov A.N., Genina E.A., Sinichkin Yu.P., Lakodina N.A. Pis'ma Zh. Tekh. Fiz., 27, 10 (2001).

12. Tuchin V.V., Maksimova I.L., Zimnyakov D.A., Kon I.L., Mavlutov A.H., Mishin A.A. J. Biomed. Opt., 2, 401 (1997).

13. Wang R.K., Xu X., Tuchin V.V., Elder J.B. J. Opt. Soc. Am. B, 18, 948 (2001).

14. Brezinski M., Saunders K., Jesser C., Li X.D., Fujimoto J. Circulation, 103, 1999 (2001).

15. Tuchin V.V., Xu X., Wang R.K. Appl. Opt., 41, 258 (2002).

16. Tuchin V.V., Utz S.R., Yaroslavsky I.V. Opt. Eng., 33, 3178 (1994).

17. Tsai C.-L., Yang Y.-F., Han C.-C., Hsieh J.-H., Chang M. Appl. Opt., 40, 5770 (2001).

18. Hiraoka M., Firbank M., Essenpreis M., Cope M., Arridge S.R., van der Zee P., Delpy D.T. Phys. Med. Biol., 38, 1859 (1993).

19. Arridge S. Appl. Opt., 34, 7395 (1995); Arridge S., Schweiger M. Appl. Opt., 34, 8026 (1995).

20. Firbank M., Okada E., Delpy D.T. Neuroimage, 8, 69 (1998).

21. Maier J. S., Gratton E. Proc. SPIE Int. Soc. Opt. Eng., 1888, 440 (1993).

22. Sevick E.M., Burch C.L., Frisoli J.K., Lakowicz J.R. Appl. Opt., 33, 3562 (1994).

23. Schotland J.C., Haselgrove J.C., Leigh J.S. Appl. Opt., 32, 448 (1993).

24. Okada E., Firbank M., Delpy D.T. Phys. Med. Biol., 40, 2093 (1995).

25. Kumar G., Schmitt J.M. Appl. Opt., 36, 2286 (1997).

26. Ermakov S.M., Mikhailov G.A. Statisticheskoe modelirovanie (Statistical Modeling) (Moscow: Nauka, 1982).

27. Marchuk G.I., Mikhailov G.A., Nazaraliev M.A., Darbinjan R.A., Kargin B.A., Elepov B.S. The Monte Carlo Methods in Atmospheric Optics (Berlin: Springer, 1980).

28. Bonner R.F., Nossal R., Havlin S., Weiss G.H. J. Opt. Soc. Am. A, 4, 423 (1987).

29. Meglinskii I.V., Matcher S.J. Opt. Spektrosk., 91, 692 (2001).

30. Meglinsky I.V., Matcher S.J. Med. Biol. \& Eng. Comput., 39, 44 (2001).

31. Odland G.F., in Physiology, Biochemistry, and Molecular Biology of the Skin. Ed. by L.A.Goldsmith (Oxford: Oxford Univ. Press, 1991) Vol. 1, pp 3 - 62.

32. Chernukh A.M., Frolov E.P. Kozha (stroenie, funktsiya, obshchaya patologiya, terapiya) (Skin: Structure, Function, General Pathology, Therapy) (Moscow: Meditsina, 1982) p. 336.

33. Lu J.Q., Hu X.-H., Dong K. Appl. Opt., 39, 5890 (2000).

34. Yaroslavskii I.V., Tuchin V.V. Opt. Spektrosk., 72, 934 (1992).

35. Keijzer M., Jaques S.L., Prahl S.A., Welch A.J. Laser Surg. Med., 9, 148 (1989).

36. Wang L., Jacques S.L., Zheng L. Computer Methods and Programs in Biomedicine, 47, 131 (1995).

37. Sobol' I.M. Metod Monte-Karlo (Monte Carlo Method) (Moscow: Nauka, 1985) p. 80.

38. Schmitt J.M., Ben-Letaief K. J. Opt. Soc. Am. A, 13, 952 (1996).

39. Dunn A.K., Smithpeter C., Welch A.J., Richards-Kortum R. Appl. Opt., 35, 3441 (1996).

40. Meglinskii I.V. Kvantovaya Elektron., 31, 1101 (2001) [Quantum Electron., 31, 1101 (2001)]. 
41. Blank I.H., Moloney J., Emslie A.G., Simon I., Apt C. J. Investigative Dermatology, 82, 188 (1984).

42. Tuchin V.V., Bashkatov A.N., Genina E.A., Kochubey V.I., Lakodina N.A., Simonenko G.V., Sinichkin Y.P., Proshina Y.M., Razumikhina N.A. Proc. SPIE Int. Soc. Opt. Eng., 3863, 10 (1999).

43. Genina E.A., Bashkatov A.N., Sinichkin Y.P., Lakodina N.A., Korovina I.V., Simonenko G.V., Tuchin V.V. Proc. SPIE Int. Soc. Opt. Eng., 4432, 97 (2001).

44. Ioffe B.V. Refraktometricheskie metody khimii (Refractometric Methods of Chemistry) (Leningrad: Khimiya, 1974) p. 400.

45. Bohren C., Huffman D. Absorption and Scattering of Light by Small Particles (New York: Wiley, 1983; Moscow: Mir, 1986).

46. Lankenau E., Welzel J., Birngruber R., Engelhardt R. Proc. SPIE Int. Soc. Opt. Eng., 2981, 78 (1997). 